\title{
Extended nonlinear Schrödinger equation with higher-order odd and even terms and its rogue wave solutions
}

\author{
Adrian Ankiewicz, ${ }^{1}$ Yan Wang, ${ }_{1}^{1}$ Stefan Wabnitz, ${ }^{2}$ and Nail Akhmediev ${ }^{1}$ \\ ${ }^{1}$ Optical Sciences Group, Research School of Physics and Engineering, The Australian National University, Canberra ACT 0200, Australia \\ ${ }^{2}$ Dipartimento di Ingegneria dell' Informazione, Università di Brescia, via Branze 38, 25123 Brescia, Italy
}

(Received 4 October 2013; revised manuscript received 15 November 2013; published 9 January 2014)

\begin{abstract}
We consider an extended nonlinear Schrödinger equation with higher-order odd (third order) and even (fourth order) terms with variable coefficients. The resulting equation has soliton solutions and approximate rogue wave solutions. We present these solutions up to second order. Moreover, specific constraints on the parameters of higher-order terms provide integrability of the resulting equation, providing a corresponding Lax pair. Particular cases of this equation are the Hirota and the Lakshmanan-Porsezian-Daniel equations. The resulting integrable equation admits exact rogue wave solutions. In particular cases, mentioned above, these solutions are reduced to the rogue wave solutions of the corresponding equations.
\end{abstract}

DOI: 10.1103/PhysRevE.89.012907

PACS number(s): 05.45.Yv, 42.65.Tg, 42.81.Qb

\section{BACKGROUND}

Wave evolution in deep water [1] and in optical fibers [2], to the lowest-order approximation, can be well described by the nonlinear Schrödinger equation (NLSE). Moreover, the NLSE is a generic equation for a multiplicity of nonlinear wave phenomena in physics, including plasma waves [3], Bose-Einstein condensates [4,5] and several other cases. The integrability of this equation [6] allows us to obtain fundamental solutions such as solitons and rogue waves in analytic form. However, the NLSE includes only basic effects on waves such as lowest-order dispersion and lowest-order nonlinearity. Higher-order effects are becoming increasingly important [7-9] when the characteristics of the solution extend beyond the simple approximations which are used in deriving the NLSE.

Higher-order terms usually have a specific structure [10] when the generalized equation is written in differential form. Particular coefficients for each term may vary and depend on experimental conditions. Attempts to find integrable cases when varying these coefficients produced several special cases such as the Hirota [11], Sasa-Satsuma [12], and LakshmananPorsezian-Daniel (LPD) [13] equations. These have been shown to be integrable, thus retaining the advantage of having solutions in analytic form. Thus, there are several exceptional cases which are integrable, and each new case is of great importance in our attempts to extend the nonlinear wave modeling beyond the fundamental NLSE case. The question arises whether these equations can be further generalized to include more of the terms considered in these particular cases.

In this work, we analyze an extended NLSE with higherorder terms and find that this equation can also be integrable, thus providing even more complicated solutions than each of the simpler equations mentioned above. Moreover, this complex equation admits a certain freedom in varying the coefficients of higher-order terms. These additional degrees of freedom may allow a more accurate adjustment of the equation to practical needs. Such adjustment also allows us to consider the known equations mentioned above as particular cases of the general equation. Additional parameters appear explicitly in the solutions of these equations making them multiparameter families of solutions. As such, they can be useful in comparisons of theoretical results with experimental data of wave evolution.

The importance of including higher-order terms has been considered in fiber optics $[7,8]$, in the case of the Heisenberg spin chain [13], and for ocean waves [14,15]. The mathematical form of these terms is usually well determined no matter which field we consider. However, particular coefficients for each term differ. They depend on the design of the fiber or on the water depth in the case of the ocean waves. At the same time, having analytical solutions for specific cases may help in comparisons with experiment when these coefficients are close to those in practice. Thus, presenting new integrable cases and their solutions provides a bridge between the theory and experiment.

\section{GENERALIZED EQUATION}

In this paper we consider the following equation which includes higher-order even and odd terms:

$$
\begin{aligned}
& i \psi_{x}+\frac{1}{2} \psi_{t t}+|\psi|^{2} \psi \\
& \quad+\gamma\left(\psi_{t t t t}+6 r_{1} \psi_{t}^{2} \psi^{*}+4 r_{2} \psi\left|\psi_{t}\right|^{2}\right. \\
& \left.\quad+8 r_{3} \psi_{t t}|\psi|^{2}+2 r_{4} \psi_{t t}^{*} \psi^{2}+6 r_{5} \psi|\psi|^{4}\right) \\
& \quad-i \alpha_{3}\left(\psi_{t t t}+6 r_{6} \psi_{t}|\psi|^{2}+6 r_{7} \psi^{2} \psi_{t}^{*}\right)=0,
\end{aligned}
$$

where $x$ is the propagation variable and $t$ is time in the moving frame, with the function $\psi(x, t)$ being the envelope of the waves. Seven real constants $r_{j}, j=1, \ldots, 7$, are free parameters in our problem. Now, the coefficients $\alpha_{3}$ and $\gamma$ are also real parameters that control independently the values of third-order dispersion $\psi_{t t t}$, and that of fourth-order dispersion $\psi_{t t t t}$.

Equation (1) contains several particular cases. First, taking $\alpha_{3}=\gamma=0$ returns us to the standard focusing NLSE [16]:

$$
i \frac{\partial \psi}{\partial x}+\frac{1}{2} \frac{\partial^{2} \psi}{\partial t^{2}}+|\psi|^{2} \psi=0
$$

which has been a traditional basic model for nonlinear wave studies. Second, the (integrable) Hirota equation [11,17] is 
obtained when $\gamma=0, r_{6}=1$, and $r_{7}=0$ :

$$
i \psi_{x}+\frac{1}{2} \psi_{t t}+|\psi|^{2} \psi-i \alpha_{3}\left(\psi_{t t t}+6 \psi_{t}|\psi|^{2}\right)=0 .
$$

The Sasa-Satsuma equation [12] is obtained when $\gamma=0, r_{6}=$ $3 / 2$, and $r_{7}=1 / 2$ :

$$
\begin{aligned}
& i \psi_{x}+\frac{1}{2} \psi_{t t}+|\psi|^{2} \psi \\
& \quad-i \alpha_{3}\left(\psi_{t t t}+9 \psi_{t}|\psi|^{2}+3 \psi^{2} \psi_{t}^{*}\right)=0 .
\end{aligned}
$$

The LPD equation [13] corresponds to the case $\alpha_{3}=0$ and $r_{j}=1$ for $j=1, \ldots, 5$ :

$$
\begin{aligned}
& i \psi_{x}+\frac{1}{2} \psi_{t t}+|\psi|^{2} \psi \\
& \quad+\gamma\left(\psi_{t t t t}+6 \psi_{t}^{2} \psi^{*}+4 \psi\left|\psi_{t}\right|^{2}\right. \\
& \left.\quad+8 \psi_{t t}|\psi|^{2}+2 \psi_{t t}^{*} \psi^{2}+6 \psi|\psi|^{4}\right)=0 .
\end{aligned}
$$

This equation has been found as a continuum limit of the Heisenberg spin chain and features integer resonance values in the Painlevé test. In optical waveguide contexts [16], the terms describe fourth-order dispersion, quintic nonlinearity, etc. It has been shown to be integrable [18]. This equation can be derived from certain moving helical curves in space using the Serret-Frenet formalism [19]. Its set of conserved quantities has been listed in [20].

Thus, containing several integrable cases, the more general equation (1) deserves detailed studies. The equation may also be integrable, and establishing this fact could help in making further progress. However, our main aim here is to find out whether this equation has rogue wave solutions. If such solutions do exist, they have to contain previously known solutions as particular cases.

In our earlier works [21,22], we extended the NLSE and included into consideration a self-steepening term $\sim s_{a} \frac{\partial}{\partial t}\left(|\psi|^{2} \psi\right)$, a term related to the self-frequency shift $\sim a_{f} \psi \frac{\partial}{\partial t}\left(|\psi|^{2}\right)$, and a third-order dispersion term $\psi_{t t t}$, as perturbations. Our analysis showed that rogue waves can certainly "survive" some perturbing effects. By comparing equations studied in [21] and [22] [Eq. (2) with $s=\alpha_{3}$ ] with Eq. (1) here, we can see that the previous cases correspond to $\gamma=0$ with $r_{6}=-\left(a_{f}+2 s_{a}\right) / 6$ and $r_{7}=-\left(a_{f}+s_{a}\right) / 6$, so that $a_{f}=6\left(r_{6}-2 r_{7}\right)$ and $s_{a}=6\left(r_{7}-r_{6}\right)$. The third-order dispersion is the same in both equations $\left(\gamma_{3}=1\right)$. We start our analysis with checking if Eq. (1) has soliton solutions.

\section{SOLITON SOLUTIONS}

Concentrating only on the simplest cases, let us take the solution in the form

$$
\psi=c \operatorname{sech}\left[c\left(t+c^{2} \alpha_{3} x\right)\right] \exp \left[\frac{i}{2} c^{2}\left(1+2 c^{2} \gamma\right) x\right],
$$

which is the fundamental zero-velocity soliton solution of Eq. (1). The coefficients $\left(c, \alpha_{3}, \gamma, r_{1}, r_{2}, r_{3}, r_{6}\right)$ are arbitrary, so long as

$$
\begin{gathered}
r_{4}=2\left(5-r_{2}-2 r_{3}\right)-3 r_{1}, \\
r_{5}=\frac{1}{3}\left(8-3 r_{1}-2 r_{2}\right), \\
r_{7}=1-r_{6} .
\end{gathered}
$$

It is easy to check that for these conditions, Eq. (4) is indeed the solution of (1).

We can also allow both the amplitude and the phase front to be at angles to the propagation axis, and thus find the solution more general than (4):

$$
\psi=c \operatorname{sech}\left[c\left(t+v_{m} x\right)\right] \exp \left[i\left(k t+r_{m} x\right)\right],
$$

with velocity

$$
v_{m}=4 \gamma k\left(k^{2}-c^{2}\right)-k+\alpha_{3}\left(c^{2}-3 k^{2}\right)
$$

and propagation constant

$$
\begin{aligned}
r_{m}= & \frac{c^{2}-k^{2}}{2}+\gamma\left(c^{2}-2 c k-k^{2}\right)\left(c^{2}+2 c k-k^{2}\right) \\
& +k \alpha_{3}\left(3 c^{2}-k^{2}\right) .
\end{aligned}
$$

Solution (8) is valid for arbitrary real numbers $\left(c, \alpha_{3}, \gamma, k, r_{1}, r_{2}, r_{3}, r_{4}\right)$. The constraints on three other parameters are

$$
\begin{gathered}
r_{5}=r_{1}+\frac{2}{3}\left(r_{2}+4 r_{3}+r_{4}-6\right), \\
r_{6}=1+\frac{\gamma}{6 k \alpha_{3}}\left[k^{2}\left(9 r_{1}-2\left(9+r_{2}-6 r_{3}\right)-r_{4}\right)\right. \\
\left.-c^{2}\left(3 r_{1}+2 r_{2}+4 r_{3}+r_{4}-10\right)\right], \\
r_{7}=\frac{\gamma}{6 k \alpha_{3}}\left[k^{2}\left(3 r_{1}+2 r_{2}+4 r_{3}-3 r_{4}-6\right)\right. \\
\left.+c^{2}\left(3 r_{1}+2 r_{2}+4 r_{3}+r_{4}-10\right)\right] .
\end{gathered}
$$

For the particular case of the Sasa-Satsuma equation $[12,23,24]\left(\gamma=0, r_{6}=3 / 2, r_{7}=1 / 2, \alpha_{3} \neq 0\right)$, we have the simplest moving soliton with fixed velocity

$$
\begin{aligned}
\psi= & c \operatorname{sech}\left(\frac{c\left[12 \alpha_{3}\left(2 c^{2} \alpha_{3} x+t\right)+x\right]}{6 \sqrt{2} \alpha_{3}}\right) \\
& \times \exp \left[-\frac{i\left(18 \alpha_{3} t+x\right)}{108 \alpha_{3}^{2}}\right],
\end{aligned}
$$

and arbitrary amplitude $c$.

For the equation combining the LPD equation with the Sasa-Satsuma equation ( $\gamma$ arbitrary, $r_{6}=3 / 2, r_{7}=1 / 2$ ), we have a stationary soliton

$\psi=c \operatorname{sech}\left[\sqrt{2} c\left(t+2 c^{2} \alpha_{3} x\right)\right] \exp \left[i c^{2}\left(1+4 c^{2} \gamma\right) x\right]$

as the basic soliton solution, with $\left(c, \alpha_{3}, \gamma, r_{1}, r_{2}, r_{3}\right)$ arbitrary, so long as $\gamma \neq 0$ and

$$
\begin{gathered}
r_{4}=\frac{1}{4 c^{2} \gamma}-3 r_{1}-2 r_{2}-4 r_{3}+20, \\
r_{5}=\frac{1}{3 c^{2} \gamma}-2 r_{1}-\frac{4 r_{2}}{3}+\frac{32}{3} .
\end{gathered}
$$

Obviously, there are more involved solutions than the ones presented above. They can be found, for example, in [12,25-27]. However, our aim here is just to show that certain types of solutions can be obtained without making use of the inverse-scattering technique and being involved in integrability issues. In a similar fashion, we now move to the simplest rogue wave solutions. 


\section{ROGUE WAVE SOLUTIONS}

Rogue waves are doubly localized solutions on a background. They can be water waves on the ocean, plasma disturbances [28], or bright light pulses propagating in optical fibers [29]. Their maximum amplitudes can be much higher than the level of the background. Therefore, they can be considered as prototypes of rogue waves in the ocean [30]. For the case $r_{j}=1, j=1, \ldots, 6$ and $r_{7}=0$, which is a combination of Hirota and LPD equations, we find the following rogue wave solution, with $c$ being the background level:

$$
\psi(x, t)=c\left[-1+4 \frac{1+2 i c^{2} B x}{D(x, t)}\right] e^{i(F x+n t)},
$$

where

$$
D(x, t)=1+4 B^{2} c^{4} x^{2}+4 c^{2}(t-v x)^{2},
$$

and

$$
B=1+12 \gamma\left(c^{2}-n^{2}\right)+6 n \alpha_{3} .
$$

with an arbitrary real parameter $n$.

Thus, $B$ here acts as a stretching factor on $x$, since, apart from the phase term, we have $x \rightarrow c^{2} B x$ relative to the rogue wave solution of the NLSE [31]. The coefficient $F$ in the exponential factor is equal to

$$
F=c^{2}-\frac{n^{2}}{2}+\gamma\left(6 c^{4}-12 c^{2} n^{2}+n^{4}\right)+n \alpha_{3}\left(6 c^{2}-n^{2}\right),
$$

and the velocity is

$$
v=n+4 n \gamma\left(6 c^{2}-n^{2}\right)+3 \alpha_{3}\left(n^{2}-2 c^{2}\right) .
$$

These expressions define the simplest rogue wave solution of our extended NLSE. Despite the simplicity, this solution has four arbitrary real parameters: $\left(c, \alpha_{3}, \gamma, n\right)$. One case of the rogue wave solution is shown in Fig. 1. The maximum of $|\psi(x, t)|$ occurs at the origin, where $\psi(0,0)=3 c$ just as in the case of the Peregrine breather. When $\gamma=0$, this solution reduces to the Hirota rogue wave [17], while it reduces to the LPD rogue wave [32] when $\alpha_{3}=0$. The rogue wave of the Sasa-Satsuma equation is much more involved [23,24]

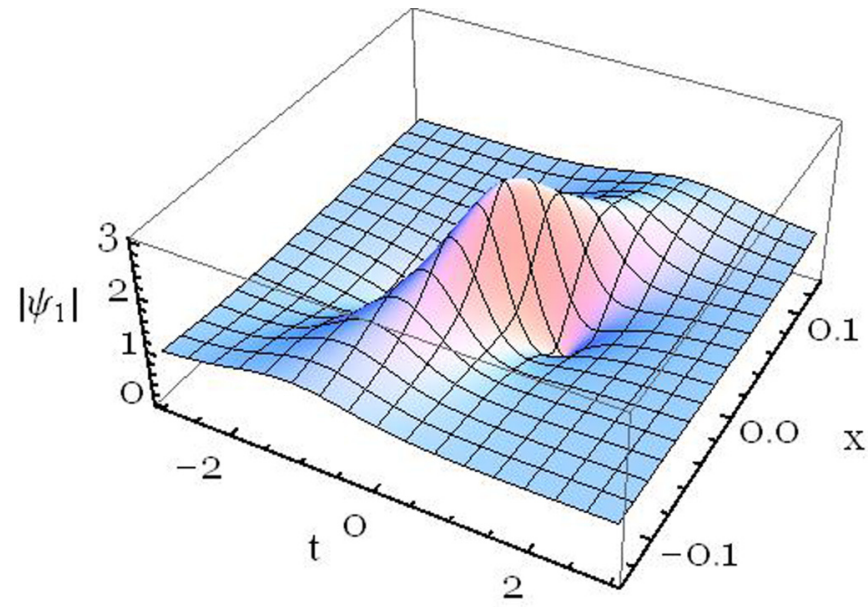

FIG. 1. (Color online) First-order rogue wave of Eq. (1), viz., Eq. (16). Here $n=0.6, \alpha_{3}=1, \gamma=1, c=1.0$. and cannot be expressed in such simple form as above. Such analysis is beyond our aim in the present study.

\section{PERTURBED ROGUE WAVE SOLUTIONS}

\section{A. Perturbation on LPD part}

For convenience, we take a unit background, $c=1$. If we take $\gamma$ to be small with $\alpha_{3}=0$ in Eq. (1), we can find an approximate solution which is valid with some arbitrary equation parameters. Here we take $\left(r_{1}, r_{3}\right)$ arbitrary, with $r_{2}=3-2 r_{3}, r_{4}=\frac{1}{2}\left(9-3 r_{1}-4 r_{3}\right), r_{5}=1$, and obtain

$$
\begin{aligned}
\psi(x, t) \approx & {\left[-1+\frac{4}{D_{1}}[1+2 i x(1+12 \gamma)]\right.} \\
& \left.+\frac{\gamma}{D_{0}^{2}}\left(R_{1}+i x J_{1}\right)+\cdots\right] e^{i x(1+6 \gamma)},
\end{aligned}
$$

where

$$
\begin{aligned}
R_{1}= & -24\left[3+r_{1}-4 r_{3}+8 t^{2}\left(r_{1}-1\right)\right. \\
& \left.+12 x^{2}\left(4 r_{3}+3 r_{1}-7\right)\right]
\end{aligned}
$$

and

$$
\begin{aligned}
J_{1}= & -48\left[17-5 r_{1}-12 r_{3}+8 t^{2}\left(r_{1}-1\right)\right. \\
& \left.+4 x^{2}\left(4 r_{3}+3 r_{1}-7\right)\right],
\end{aligned}
$$

correct to order $\gamma$.

We are using the case $\left(c=1, n=0, \alpha_{3}=0\right)$ of $D(x, t)$ from Eq. (17) to define $D_{1}=1+4 t^{2}+4 x^{2}(1+12 \gamma)^{2}$, while the Peregrine case $(\gamma=0)$ is denoted $D_{0}=1+4 t^{2}+4 x^{2}$. If $r_{1}=1$ and $r_{3}=1$, then $R_{1}=0$ and $J_{1}=0$, so Eq. (18) reduces to Eq. (16) with $\left(c=1, n=0, \alpha_{3}=0\right)$. Of course, the exact solution, Eq. (16), for $r_{j}=1, j=1, \ldots, 5$, can be expanded in $\gamma$ as

$$
\begin{aligned}
\psi(x, t) e^{-i x(1+6 \gamma) \approx} & -1+\frac{4}{D_{0}}(1+2 i x)+\frac{96 \gamma x}{D_{0}^{2}}[-4 x \\
& \left.+i\left(1+4 t^{2}-4 x^{2}\right)\right]+\cdots
\end{aligned}
$$

so we can express solution (18) as

$$
\begin{aligned}
\psi(x, t) \approx & {\left[-1+\frac{4}{D_{0}}(1+2 i x)\right.} \\
& \left.+\frac{\gamma}{D_{0}^{2}}\left(R_{0}+i x J_{0}\right)+\cdots\right] e^{i x(1+6 \gamma)},
\end{aligned}
$$

where

$$
\begin{aligned}
R_{0}= & -24\left[3+r_{1}-4 r_{3}+8 t^{2}\left(r_{1}-1\right)\right. \\
& \left.+4 x^{2}\left(9 r_{1}+12 r_{3}-17\right)\right]
\end{aligned}
$$

and

$$
\begin{aligned}
J_{0}= & -48\left[15-5 r_{1}-12 r_{3}+8 t^{2}\left(r_{1}-2\right)\right. \\
& \left.+4 x^{2}\left(4 r_{3}+3 r_{1}-5\right)\right] .
\end{aligned}
$$

Note that the perturbation part of the solution is even in $t$, reflecting the symmetry of the original equation. Indeed, the symmetry is the same as that considered in [33], which corresponds to $r_{j}=0, j=1, \ldots, 4$, in Eq. (1) and $r_{5}=$ $v /(6 \gamma), \gamma=-h v$. If $r_{1}=1$ and $r_{3}=1$, then solution (20) reduces to (19). 


\section{B. Perturbation on Hirota part}

We now take $\gamma$ to be zero assuming $\alpha_{3}$ is small in Eq. (1), and write the approximate solution. Here we take $\left(r_{6}, r_{7}\right)$ arbitrary and obtain

$$
\begin{aligned}
\psi(x, t) e^{-i x} \approx & {\left[-1+\frac{4}{D_{s}}(1+2 i x)\right.} \\
& \left.+\frac{32 t \alpha_{3}}{D_{0}^{2}}\left[k_{n} x+i f_{e}(x, t)\right]+\cdots\right],
\end{aligned}
$$

where

$$
\begin{gathered}
D_{s}=1+4 x^{2}+4\left(t+6 \alpha_{3} x\right)^{2}, \\
k_{n}=6\left(2-2 r_{6}-r_{7}\right), \\
f_{e}(x, t)=\frac{3}{2}\left[\left(r_{6}-1\right)\left(1-4 t^{2}-12 x^{2}\right)+2 r_{7}\right],
\end{gathered}
$$

correct to order $\alpha_{3}$. Converting the notation (see Sec. II of this paper) to that of [22], Eq. (21) then agrees with the result given there.

We can extend the expressions to second order in $\alpha_{3}$ by keeping $r_{7}$ arbitrary, but fixing $r_{6}=2 r_{7}+1$, so we now have $k_{n}=-30 r_{7}$ and $f_{e}(x, t)=-6 r_{7}\left(2 t^{2}+6 x^{2}-1\right)$. Additionally, the expression

$$
\frac{\alpha_{3}^{2}}{D_{0}^{3}}\left[w_{e}(x, t)+i x w_{i}(x, t)\right]
$$

has to be added to the right-hand side of Eq. (21), where

$$
\begin{aligned}
w_{e}(x, t)= & 288 r_{7}\left[16\left(4 r_{7}-1\right) t^{4}+8 t^{2}\left\{r_{7}\left(51 x^{2}-3\right)\right.\right. \\
& \left.\left.+22 x^{2}+3\right\}-\left(4 x^{2}+1\right)\left\{\left(42 r_{7}+32\right) x^{2}+1\right\}\right],
\end{aligned}
$$

and

$$
\begin{aligned}
w_{i}(x, t)= & 576 r_{7}\left[16 r_{7} t^{4}+2 x^{2}\left\{4\left(9 r_{7}+8\right) t^{2}+3 r_{7}-2\right\}\right. \\
& \left.-108 r_{7} t^{2}-8\left(9 r_{7}+8\right) x^{4}+6 r_{7}-4 t^{2}+3\right] .
\end{aligned}
$$

In this case, a perturbation part of the solution which is an odd power of $\alpha_{3}$ will be odd in $t$, as in the works [21] and [22], because the symmetry of the Hirota-type equation differs from that of the LPD-type equation. If $r_{7}=0$ (so $\left.r_{6}=1\right)$, i.e., in the case of the Hirota equation, then we have $k_{n}=0, f_{e}(x, t)=$ $w_{e}(x, t)=w_{i}(x, t)=0$, and as expected, Eq. (21) reduces to the exact Hirota rogue wave solution.

\section{LAX PAIR FORMALISM FOR THE SPECIAL CASE OF THE COMBINED EQUATION}

The combined Hirota-LPD form of Eq. (1) with $r_{j}$ coefficients fixed ( $r_{j}=1$ except that $\left.r_{7}=0\right)$ can be written in the form

$$
\begin{aligned}
& i \psi_{x}+\frac{1}{2} \psi_{t t}+|\psi|^{2} \psi+\gamma\left(\psi_{t t t t}+6 \psi_{t}^{2} \psi^{*}\right. \\
& \left.\quad+4 \psi\left|\psi_{t}\right|^{2}+8 \psi_{t t}|\psi|^{2}+2 \psi_{t t}^{*} \psi^{2}+6 \psi|\psi|^{4}\right) \\
& \quad-i \alpha_{3}\left(\psi_{t t t}+6 \psi_{t}|\psi|^{2}\right)=0,
\end{aligned}
$$

The equation can also be written in the operator form

$$
\begin{aligned}
F[\psi(x, t)]= & S[\psi(x, t)]-i \alpha_{3} H[\psi(x, t)] \\
& +\gamma P[\psi(x, t)]=0,
\end{aligned}
$$

where

$$
S[\psi(x, t)]=i \psi_{x}+\frac{1}{2} \psi_{t t}+\psi|\psi|^{2}
$$

is the nonlinear Schrödinger operator,

$$
H[\psi(x, t)]=6 \psi_{t}|\psi|^{2}+\psi_{t t t}
$$

is the Hirota operator, and

$$
\begin{aligned}
P[\psi(x, t)]= & \psi_{t t t t}+6 \psi|\psi|^{4}+4 \psi\left|\psi_{t}^{*}\right|^{2} \\
& +8 \psi_{t t}|\psi|^{2}+6 \psi_{t}^{2} \psi^{*}+2 \psi^{2} \psi_{t t}^{*}
\end{aligned}
$$

is the LPD operator.

We previously provided a matrix analysis of the nonlinear Schrödinger equation [34] and the Hirota equation [17] in order to obtain the linear set of equations representing the integrable nonlinear equation and then used the Darboux transformation to get higher-order solutions from a seed solution. For this combined case, the condition of compatibility can be written in terms of the Lax pair

$$
\begin{aligned}
& \mathbf{R}_{\mathbf{t}}=\mathbf{L R}, \\
& \mathbf{R}_{x}=\mathbf{B R},
\end{aligned}
$$

where the vector

$$
\mathbf{R}=\left[\begin{array}{l}
r \\
s
\end{array}\right]
$$

consists of two linear complex functions $r=r(x, t)$ and $s=$ $s(x, t)$ while the matrix operators

$$
\mathbf{L}=\lambda \mathbf{J}+\mathbf{U}
$$

and

$\mathbf{B}=\lambda^{2} \mathbf{J}(\mathbf{x}, \mathbf{t})+\lambda \mathbf{U}(\mathbf{x}, \mathbf{t})+\frac{1}{2} \mathbf{V}(\mathbf{x}, \mathbf{t})-\alpha_{3} \mathbf{M}(\mathbf{x}, \mathbf{t})+\gamma \mathbf{V}_{\mathbf{p}}(\mathbf{x}, \mathbf{t})$

can be written in terms of the following square matrices:

$$
\begin{aligned}
& \mathbf{U}=\left[\begin{array}{cc}
0 & i \psi^{*}(x, t) \\
i \psi(x, t) & 0
\end{array}\right], \\
& \mathbf{J}=\left[\begin{array}{cc}
i & 0 \\
0 & -i
\end{array}\right] .
\end{aligned}
$$

and

$$
\mathbf{V}=\left[\begin{array}{cc}
-i|\psi|^{2} & \psi_{t}^{*} \\
-\psi_{t} & i|\psi|^{2}
\end{array}\right],
$$

For arbitrary complex $\lambda$, we can define the operator $\mathbf{M}$ as

$$
\mathbf{M}=4 \lambda^{3} \mathbf{J}(\mathbf{x}, \mathbf{t})+4 \lambda^{2} \mathbf{U}(\mathbf{x}, \mathbf{t})+2 \lambda \mathbf{V}(\mathbf{x}, \mathbf{t})+\mathbf{K}_{\mathbf{a}}(\mathbf{x}, \mathbf{t}),
$$

where

$$
\mathbf{K}_{\mathbf{a}}=\left[\begin{array}{cc}
A_{n}(x, t) & -B_{n}(x, t)^{*} \\
B_{n}(x, t) & -A_{n}(x, t)
\end{array}\right],
$$

with

$$
\begin{gathered}
A_{n}(x, t)=\psi^{*} \psi_{t}-\psi \psi_{t}^{*} \\
B_{n}(x, t)=-i\left(2 \psi|\psi|^{2}+\psi_{t t}\right) .
\end{gathered}
$$


In our notation, the last matrix is

$$
\mathbf{V}_{\mathbf{p}}=\left[\begin{array}{cc}
i A_{p}(x, t) & B_{p}(x, t) \\
-C_{p}(x, t) & i D_{p}(x, t)
\end{array}\right],
$$

where

$$
\begin{aligned}
D_{p}(x, t)= & 3|\psi|^{4}-\left|\psi_{t}\right|^{2}+2 i \lambda\left(\psi \psi_{t}^{*}-\psi^{*} \psi_{t}\right) \\
& +\psi^{*} \psi_{t t}+\psi \psi_{t t}^{*}-4 \lambda^{2}|\psi|^{2}+k_{2}+8 \lambda^{4},
\end{aligned}
$$

and

$$
A_{p}(x, t)=-D_{p}(x, t)
$$

Further,

$$
\begin{aligned}
B_{p}(x, t)= & 2 \psi^{*}\left(3 \psi \psi_{t}^{*}-4 i \lambda^{3}\right)+2 i \lambda \psi_{t t}^{*}+\psi_{t t t}^{*} \\
& +4 i \lambda \psi^{*}|\psi|^{2}-4 \lambda^{2} \psi_{t}^{*},
\end{aligned}
$$

and

$$
\begin{aligned}
C_{p}(x, t)= & 2 \psi\left(3 \psi^{*} \psi_{t}+4 i \lambda^{3}\right)-2 i \lambda \psi_{t t}+\psi_{t t t} \\
& -4 i \lambda \psi|\psi|^{2}-4 \lambda^{2} \psi_{t} .
\end{aligned}
$$

Note that $B_{p}(x, t)=C_{p}(x, t)^{*}$ if $\lambda$ is real. Clearly, the linear functions $r$ and $s$ depend on which solution $\psi(x, t)$ we use in the matrices. form

Now the condition of compatibility $\mathbf{R}_{t x}=\mathbf{R}_{x t}$ takes the

$$
\mathbf{B} \mathbf{L}-\mathbf{L} \mathbf{B}+\mathbf{B}_{t}-\mathbf{L}_{x}=0 .
$$

Expanding the left-hand side gives

$$
\left[\begin{array}{cc}
0 & F^{*}[\psi(x, y)] \\
-F[\psi(x, y)] & 0
\end{array}\right],
$$

where $F[\psi(x, y)]$ is defined in Eq. (26). Clearly, this is identically zero for any valid solution of Eq. (25), because $F[\psi(x, y)]=0$. Equation (36) is correct for all $\lambda, \alpha_{3}$, and $\gamma$. Thus, we have demonstrated the existence of the Lax pair and the spectral parameter $\lambda$ for the system of Eq. (25).

The integrability of the Hirota-LPD equation (25) should not be confused with the asymptotic integrability considered in Refs. [36,37]. Our extended equation is integrable for arbitrary values of the two independent parameters $\gamma$ and $\alpha_{3}$ controlling higher-order terms, while the equations in [36,37] are only "nearly integrable" at small values of the perturbation.

\section{GENERATING SOLUTIONS}

Solutions of the linear equations in $r, s$ can be used to generate higher-order solutions of the nonlinear equation. Equation (27) provides the linear equations (here we choose $\lambda=i$, since we are interested in rogue waves). In this case

$$
\mathbf{M}=\left[\begin{array}{cc}
a_{1} & -i a_{3} \\
-i a_{2} & -a_{1}
\end{array}\right]
$$

where

$$
\begin{gathered}
a_{1}=4+2|\psi|^{2}-\psi \psi_{t}^{*}+\psi^{*} \psi_{t}, \\
a_{2}=f+2 \psi_{t}, \\
a_{3}=f^{*}-2 \psi_{t}^{*},
\end{gathered}
$$

and the functional

$$
f[\psi]=4 \psi+2 \psi|\psi|^{2}+\psi_{t t}
$$

Then

$$
\mathbf{R}_{\mathbf{t}}=\left[\begin{array}{c}
r_{t} \\
s_{t}
\end{array}\right]=\left[\begin{array}{c}
i \psi^{*} s-r \\
i \psi r+s,
\end{array}\right]
$$

while

$$
\mathbf{R}_{\mathbf{x}}=\left[\begin{array}{c}
r_{x} \\
s_{x}
\end{array}\right]=\frac{i}{2}\left[\begin{array}{l}
r m_{1}+s m_{2} \\
r m_{3}+s m_{4}
\end{array}\right]
$$

where the functions $m_{1}, m_{2}, m_{3}$, and $m_{4}$ are given in Appendix A. When $\alpha_{3}=\gamma=0$, the above set of equations is reduced to the linear set presented in [34] for the choice of the parameter $l=i=\sqrt{-1}$. It corresponds to the NLSE.

We can now solve the linear equations (43) and (44) for some seed solution $\psi_{0}$. To be specific, we choose the plane wave solution $\psi_{0}=e^{i(1+6 \gamma) x}$. We obtain

$$
r=\frac{\left(-1+2 t+12 \alpha_{3} x+2 i B x\right)}{\sqrt{2}} \exp \left[-\frac{i x}{2}(6 \gamma+1)\right]
$$

and

$$
s=-\frac{i}{\sqrt{2}}\left(1+2 t+12 \alpha_{3} x+2 i B x\right) \exp \left[\frac{i x}{2}(6 \gamma+1)\right] \text {. }
$$

We can rewrite these solutions in the form

$$
\begin{aligned}
& r=\sqrt{2}\left(-\frac{1}{2}+t-v x+i B x\right) \sqrt{\psi_{0}^{*}} \\
& s=-i \sqrt{2}\left(\frac{1}{2}+t-v x+i B x\right) \sqrt{\psi_{0}}
\end{aligned}
$$

where $B=(1+12 \gamma)$ and $v=-6 \alpha_{3}$. Thus, the product $s r^{*}$ is proportional to $\psi_{0}$. These linear functions reduce to the functions found in the case of the Hirota equation [17] when $\gamma=0$, and the NLS case when $\alpha_{3}=\gamma=0$.

The new, higher-order solution $\psi_{1}$ is found from

$$
\begin{aligned}
\psi_{1} & =\psi_{0}-\frac{4 i s r^{*}}{\left(|r|^{2}+|s|^{2}\right)} \\
& =-\psi_{0}+\frac{4(1+2 i x B) \psi_{0}}{1+4(t-v x)^{2}+4 B^{2} x^{2}} .
\end{aligned}
$$

This agrees with Eq. (16) given earlier for the ( $c=1, n=0)$ case. So far, the analysis was quite general. Now, we can proceed further and find the second-order solution for the combined equation. 


\section{SECOND-ORDER ROGUE WAVE SOLUTIONS}

\section{A. LPD equation}

For the LPD equation when $\alpha_{3}=0$ and $r_{j}=1$ for $j=1, \ldots, 5$, we find the linear functions to be

$$
\begin{aligned}
r_{2}(x, t)= & \frac{e^{-i(1+6 \gamma) x / 2}}{D_{p} \sqrt{2}}\left\{16 B^{4} x^{4}\right. \\
& -32 i B^{3}(t-1) x^{3}-8 i x\left[B\left(4 t^{3}-7 t-5\right)+4 t+2\right] \\
& \left.+8 B x^{2}[B(6 t+7)-4]-16 t^{4}+16 t^{3}+12 t-3\right\},
\end{aligned}
$$

$$
\begin{aligned}
s_{2}(x, t)= & -\frac{i e^{i(1+6 \gamma) x / 2}}{D_{p} \sqrt{2}}\left\{-16 B^{4} x^{4}\right. \\
& +32 i B^{3}(t+1) x^{3}+8 i x\left[B\left(4 t^{3}-7 t+5\right)+4 t-2\right] \\
& \left.+8 B x^{2}[B(6 t-7)+4]+16 t^{4}+16 t^{3}+12 t+3\right\},
\end{aligned}
$$

where $D_{p}=4 B^{2} x^{2}+4 t^{2}+1$, with $B=1+12 \gamma$, as before.

Hence, the second-order rogue wave [32], converted to our notation, is

$$
\psi(x, t)=\left[1+12 \frac{G_{p}+i H_{p}}{D_{p}(x, t)}\right] e^{i(1+6 \gamma) x},
$$

where where the polynomials $G_{p}, H_{p}$, and $D_{p}$ are presented in Appendix B.

When $\gamma=0$, then Eq. (52) naturally reduces to the secondorder NLSE result [34], viz.,

$$
\psi_{n}(x, t)=\left[1+12 \frac{G_{n}+i H_{n}}{D_{n}(x, t)}\right] e^{i x},
$$

where

$$
\begin{aligned}
& G_{n}=-16 t^{4}-96 t^{2} x^{2}-24 t^{2}-80 x^{4}-72 x^{2}+3, \\
& H_{n}=-2 x\left[16 t^{4}+8 t^{2}\left(4 x^{2}-3\right)+16 x^{4}+8 x^{2}-15\right],
\end{aligned}
$$

and

$$
\begin{aligned}
D_{n}= & 64 t^{6}+48 t^{4}\left(4 x^{2}+1\right)+12 t^{2}\left(3-4 x^{2}\right)^{2} \\
& +64 x^{6}+432 x^{4}+396 x^{2}+9,
\end{aligned}
$$

since $G_{p} \rightarrow G_{n}, H_{p} \rightarrow H_{n}$, and $D_{p} \rightarrow D_{n}$.

\section{B. Hirota equation}

We now present the second-order Hirota equation rogue wave [17] in compact notation. Again, the linear functions are

$$
\begin{aligned}
r_{2}(x, t)= & \frac{e^{-i x / 2}}{D_{h} \sqrt{2}}\left[4 \left(-6 x^{2}\left[24 \alpha_{3}^{2}\left(6 t^{2}-3 t-2\right)\right.\right.\right. \\
& \left.+2 i \alpha_{3}\left(12 t^{2}+1\right)-2 t-1\right] \\
& +2 t x\left\{12 \alpha_{3}[(3-4 t) t+2]-4 i t^{2}+3 i\right\} \\
& -8\left(6 \alpha_{3}+i\right)^{2} x^{3}\left[3 \alpha_{3}(4 t-1)-i(t-1)\right] \\
& \left.-4\left(6 \alpha_{3}-i\right)\left(6 \alpha_{3}+i\right)^{3} x^{4}-4 t^{4}+4 t^{3}+3 t\right) \\
& \left.+24\left(7 \alpha_{3}+i\right) x-3\right],
\end{aligned}
$$

$$
\begin{aligned}
s_{2}(x, t)= & -\frac{i e^{i x / 2}}{D_{h} \sqrt{2}}\left[2 4 x ^ { 2 } \left[24 \alpha_{3}^{2}\left(6 t^{2}+3 t-2\right)\right.\right. \\
& \left.+2 i \alpha_{3}\left(12 t^{2}+1\right)+2 t-1\right] \\
& +8 x\left(3 \alpha_{3}\{4 t[t(4 t+3)-2]+7\}+i\left(4 t^{3}-3 t+3\right)\right) \\
& +32\left(6 \alpha_{3}+i\right)^{2} x^{3}\left[3 \alpha_{3}(4 t+1)-i(t+1)\right] \\
& +16\left(6 \alpha_{3}-i\right)\left(6 \alpha_{3}+i\right)^{3} x^{4} \\
& \left.+16 t^{4}+16 t^{3}+12 t+3\right],
\end{aligned}
$$

where $D_{h}=4 x^{2}+4\left(t+6 \alpha_{3} x\right)^{2}+1$.

The resulting solution is

$$
\psi_{h}(x, t)=\left[1+12 \frac{G_{h}+i H_{h}}{D_{h}(x, t)}\right] e^{i x},
$$

where the polynomials $G_{h}, H_{h}$, and $D_{h}$ are presented in Appendix C. If $\alpha_{3}=0$ then $w$ in these polynomials is zero $(w=0)$ and Eq. (56) reduces to the second-order NLSE result, Eq. (53), since $G_{h} \rightarrow G_{n}, H_{h} \rightarrow H_{n}$, and $D_{h} \rightarrow D_{n}$.

So Eqs. (52) and (56) have the same forms, and they can be used to obtain higher-order solutions valid for any $\alpha_{3}, \gamma$. Of course, this is more involved than the combination in Eq. (16) for the first-order rogue wave, and it is the topic we address in the next section.

\section{SECOND-ORDER ROGUE WAVE SOLUTIONS OF COMBINED EQUATION}

For the general case, we take $\alpha_{3}, \gamma$ arbitrary and include into the solution two arbitrary parameters, $\beta$ and $\delta$. Now the linear functions take the form

$$
\begin{aligned}
& r_{2}=D_{\mathrm{tot}}^{-1} e^{-i x(1+6 \gamma) / 2}\left[r_{2 r}(x, t)+i r_{2 i}(x, t)\right], \\
& s_{2}=D_{\mathrm{tot}}^{-1} e^{i x(1+6 \gamma) / 2}\left[s_{2 r}(x, t)+i s_{2 i}(x, t)\right],
\end{aligned}
$$

where

$$
r_{2 r}(x, t)=s_{2 i}(-x,-t)-\frac{\beta}{8}\left(12 \alpha_{3} x+2 t+1\right)+\frac{\delta x}{4} B
$$

and

$$
r_{2 i}(x, t)=-s_{2 r}(-x,-t)+\frac{\delta}{8}\left(12 \alpha_{3} x+2 t+1\right)+\frac{\beta x}{4} B,
$$

while

$$
\begin{aligned}
s_{2 r}(x, t)= & -2 B x^{3}\left[108 \alpha_{3}^{2} t+24 \gamma(6 \gamma+1)(t+1)+t+1\right] \\
& -12 \alpha_{3} B x^{4}\left(36 \alpha_{3}^{2}+B^{2}\right) \\
& -\frac{1}{8} x\left\{6 \alpha_{3} \delta+B \beta+4\left[12 \gamma\left(4 t^{3}-7 t+5\right)\right.\right. \\
& \left.\left.+4 t^{3}-3 t+3\right]\right\}-3 \alpha_{3} x^{2}\left(12 B t^{2}-36 \gamma+1\right) \\
& +\frac{1}{16} \delta(1-2 t),
\end{aligned}
$$

and

$$
\begin{aligned}
s_{2 i}(x, t)= & \frac{1}{16}\left[2 \left(-8 x^{4}\left(B^{4}-1296 \alpha_{3}^{4}\right)\right.\right. \\
& +12 x^{2}\left\{24 \alpha_{3}^{2}\left(6 t^{2}+3 t-2\right)\right. \\
& +B[4 \gamma(6 t-7)+2 t-1]\} \\
& +144 \alpha_{3} x^{3}\left[12 \alpha_{3}^{2}(4 t+1)+B^{2}\right]
\end{aligned}
$$




$$
\begin{aligned}
& \left.+x\left\{48 \alpha_{3} t[t(4 t+3)-2]+B \delta\right\}+8 t^{3}(t+1)\right) \\
& \left.+\beta\left(-12 \alpha_{3} x-2 t+1\right)+3\left(56 \alpha_{3} x+4 t+1\right)\right] .
\end{aligned}
$$

Here, $D_{\text {tot }}$ is the $n=0, c=1$ form of Eq. (17):

$$
D_{\text {tot }}(x, t)=1+4(B x)^{2}+4\left(t+6 \alpha_{3} x\right)^{2},
$$

with $B=1+12 \gamma$.

The solution in standard form is

$$
\psi_{2}=\psi_{1}-\frac{4 i s_{2} r_{2}^{*}}{\left|r_{2}\right|^{2}+\left|s_{2}\right|^{2}},
$$

Substituting here the linear functions, we obtain the secondorder rogue wave solution of the combined equation:

$$
\psi_{2}=\left[\frac{G_{2}+i K_{2}}{D_{2}}+1\right] e^{i x(1+6 \gamma)}
$$

with the polynomials $G_{2}, K_{2}$, and $D_{2}$ given as

$$
\begin{aligned}
& G_{2}=12\left[4 \left(-288 \alpha_{3} t x^{3}\left(12 \alpha_{3}^{2}+B^{2}\right)\right.\right. \\
& -4 x^{4}\left(36 \alpha_{3}^{2}+B^{2}\right)\left(36 \alpha_{3}^{2}+5 B^{2}\right) \\
& -6 x^{2}\left\{12 \alpha_{3}^{2}\left(12 t^{2}+11\right)+B\left[4 B t^{2}+68 \gamma+3\right]\right\} \\
& +x\left\{-6 \alpha_{3}\left[\beta+4 t\left(4 t^{2}+7\right)\right]+\delta B\right\} \\
& \left.\left.-t\left(\beta+4 t^{3}+6 t\right)\right)+3\right] \text {, } \\
& K_{2}=12\left(8 x \left\{-2 x^{2}\left[36 \alpha_{3}^{2}\left(12 B t^{2}+12 \gamma+5\right)\right.\right.\right. \\
& \left.+B^{2}\left(4 B t^{2}+60 \gamma+1\right)\right] \\
& -96 \alpha_{3} B t x^{3}\left(36 \alpha_{3}^{2}+B^{2}\right)-4 B x^{4}\left(36 \alpha_{3}^{2}+B^{2}\right)^{2} \\
& -6 \alpha_{3} x\left[B \beta+4 t\left(4 B t^{2}-36 \gamma+1\right)\right] \\
& \left.-t\left[B \beta+4 B t^{3}-6(28 \gamma+1) t\right]\right\} \\
& +\delta\left\{-4\left[x\left(6 \alpha_{3}-B\right)+t\right]\left(6 \alpha_{3} x\right.\right. \\
& +12 \gamma x+t+x)-1\}+6(92 \gamma+5) x) \text {, } \\
& D_{2}=-12 x^{2}\left\{12 \alpha_{3}^{2}\left[12 t\left(\beta-20 t^{3}+10 t\right)-139\right]\right. \\
& -48 \alpha_{3} B \delta t-4 B^{2} \beta t-16 B^{2} t^{4} \\
& \left.+24 B(44 \gamma+1) t^{2}-3[8 \gamma(614 \gamma+57)+11]\right\} \\
& +2304 \alpha_{3} t x^{5}\left(36 \alpha_{3}^{2}+B^{2}\right)^{2} \\
& +64 x^{6}\left(36 \alpha_{3}^{2}+B^{2}\right)^{3}+48 x^{4}\left[432 \alpha_{3}^{4}\left(60 t^{2}-13\right)\right. \\
& \left.+72 \alpha_{3}^{2} B\left(12 B t^{2}-36 \gamma+5\right)+B^{3}\left(4 B t^{2}+172 \gamma+9\right)\right] \\
& +16 x^{3}\left\{-216 \alpha_{3}^{3}\left(\beta-80 t^{3}+36 t\right)+108 \alpha_{3}^{2} \delta B\right. \\
& \left.+18 \alpha_{3} B\left[B \beta+4 t\left(4 B t^{2}-84 \gamma+1\right)\right]-B^{3} \delta\right\} \\
& +12 x\left\{\alpha_{3}\left[2 \beta\left(11-12 t^{2}\right)+4 t\left(48 t^{4}-8 t^{2}+51\right)\right]\right. \\
& \left.+\delta\left(4 B t^{2}-68 \gamma-3\right)\right\}+\beta^{2}-16 \beta t^{3}+12 \beta t \\
& +\delta^{2}+64 t^{6}+48 t^{4}+108 t^{2}+9 \text {. }
\end{aligned}
$$

Examples of rogue waves for various sets of parameters are given in Figs. 2, 3, and 4. We note that the value of the function

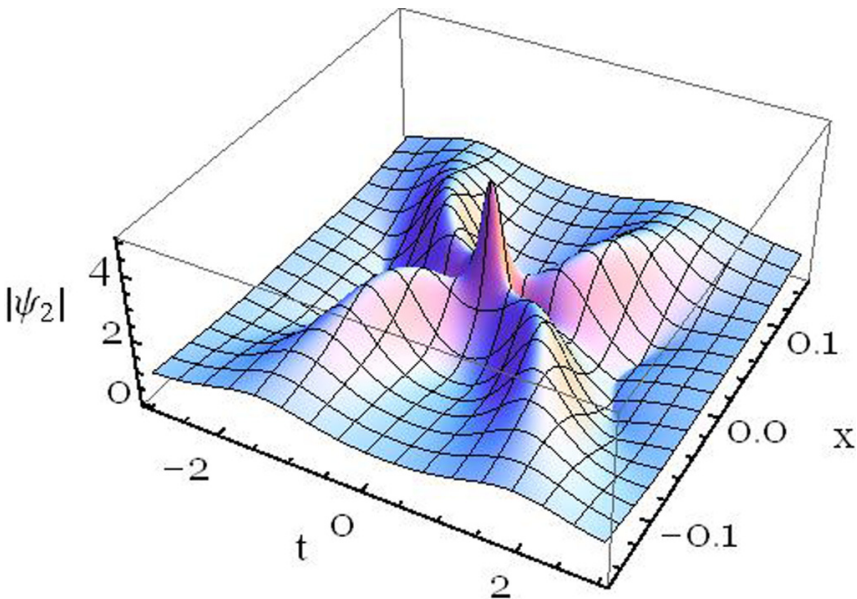

FIG. 2. (Color online) Second-order rogue wave given by Eq. (61). The parameters are $\alpha_{3}=1 / 4, \gamma=1, \beta=0$, and $\delta=0$.

at the origin is

$$
\left|\psi_{2}(0,0)\right|^{2}=\frac{2\left(\beta^{2}+117\right) \delta^{2}+\left(\beta^{2}+45\right)^{2}+\delta^{4}}{\left(\beta^{2}+\delta^{2}+9\right)^{2}} .
$$

The maximum of this function occurs when $\beta=\delta=0$. If $\delta=0$, then

$$
\psi_{2}(0,0)=1+\frac{36}{9+\beta^{2}}(\leqslant 5),
$$

so the maximum cannot be higher than that $(2 N+1=5)$ of the basic second-order $(N=2)$ NLSE rogue wave. For $\alpha_{3}=\gamma=0$, we obtain the second-order "triplet" solution of the NLSE [35] with its two arbitrary parameters. When $\beta$ and $\delta$ are not close to zero, the three fundamental waves appear on a circle in the $(x, t)$ plane and form an equilateral triangle. The circle has its center at $(0,0)$. When $\beta=\delta=0$, it is the nonlinear superposition of the three first-order rogue waves which produces the familiar high-peak second-order rogue wave.

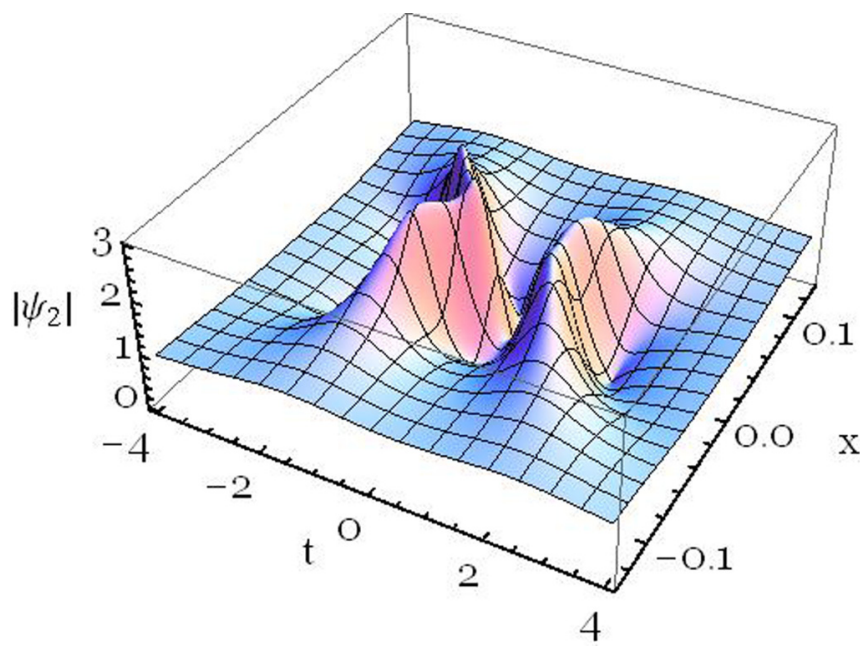

FIG. 3. (Color online) Rogue wave given by Eq. (61). The parameters are $\alpha_{3}=1 / 2, \gamma=2, \beta=10$, and $\delta=0$. The solution splits and reveals two maxima. This is an early stage of separation into a triplet. 


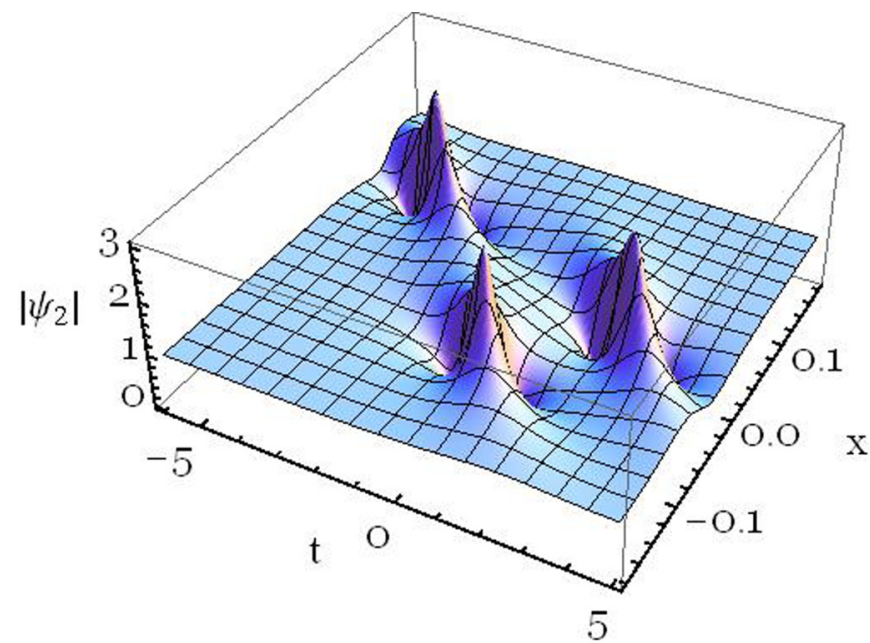

FIG. 4. (Color online) Rogue wave given by Eq. (61) where the large value of $\beta$ results in a tilted triplet. The parameters are $\alpha_{3}=4$, $\gamma=2, \beta=150$, and $\delta=0$. Note that the three components do not form an equilateral triangle centered on the origin.

\section{CONCLUSION}

In conclusion, we constructed a generalized NLS evolution equation with odd and even higher-order terms which contains as particular cases previously known integrable higher-order NLS equations. We have shown that certain combinations of higher-order terms provide integrability of the resulting higherorder NLSE. Consequently, some solutions of these equations can be written in explicit form. As particular examples, we presented explicitly rogue wave solutions of the first and second order for these equations. When coefficients are such that integrability is lifted, we still can obtain approximate rogue wave solutions of the extended NLSE with higher-order terms.

Because these terms are important in real wave propagation systems [7,8,10,13-15], each step in their study is a significant step forward. Moreover, each integrable case provides us with analytic solutions for equations beyond the simple NLSE case. Having nine higher-order terms in the extension of the NLSE allows us to describe more physical effects influencing nonlinear wave propagation than ever before. Moreover, two free parameters present in this equation provide closer adjustment to the experimental situations. As the number of NLSE extensions that are integrable can be counted using the fingers of one hand, each new case is vitally important for the description of wave phenomena with increasing accuracy. Having these special cases, we can develop new "near-integrable" approximations that cover more practically important sets of parameters than ever before. Such step-by-step progress may lead to a complete analysis of this intriguing problem for any number of higher-order terms in the nonlinear wave propagation.

\section{ACKNOWLEDGMENTS}

A.A. and N.A. acknowledge the support of the Australian Research Council (Discovery Project DP110102068) and also thank the Volkswagen Foundation for financial support.

\section{APPEDIDIX A: COEFFICIENTS USED IN EQ. (44)}

$$
\begin{aligned}
& m_{1}= 2 \psi_{t}\left[\gamma \psi_{t}^{*}+i\left(\alpha_{3}+2 i \gamma\right) \psi^{*}\right] \\
&+2 \psi\left(2 \gamma-i \alpha_{3}\right) \psi_{t}^{*}-\left(1+8 \gamma-4 i \alpha_{3}\right)\left(2+|\psi|^{2}\right) \\
&-2 \gamma \psi \psi_{t t}^{*}-2 \gamma \psi_{t t} \psi^{*}-6 \gamma|\psi|^{4} \\
& m_{2}= 2 \psi^{*}\left(-6 i \gamma \psi \psi_{t}^{*}+4 \alpha_{3}+8 i \gamma+i\right) \\
&+4\left(\alpha_{3}+2 i \gamma\right) \psi\left(\psi^{*}\right)^{2}-\left(4 \alpha_{3}+8 i \gamma+i\right) \psi_{t}^{*} \\
&+2\left(\alpha_{3}+2 i \gamma\right) \psi_{t t}^{*}-2 i \gamma \psi_{t t t}^{*}, \\
& \\
& m_{3}= \psi\left(i+4 \alpha_{3}+8 i \gamma+6 i \gamma \psi^{*} \psi_{t}\right) \\
&+2 i \gamma \psi_{t t t}+2\left(\alpha_{3}+2 i \gamma\right) \psi_{t t}+\left(i+4 \alpha_{3}+8 i \gamma\right) \psi_{t} \\
&+4\left(\alpha_{3}+2 i \gamma\right) \psi|\psi|^{2}, \\
& m_{4}=2 i \psi_{t}\left[i \gamma \psi_{t}^{*}-\left(\alpha_{3}+2 i \gamma\right) \psi^{*}\right]+2 \gamma \psi \psi_{t t}^{*}+2 \gamma \psi_{t t} \psi^{*} \\
&+6 \gamma|\psi|^{4}+2 i\left(\alpha_{3}+2 i \gamma\right) \psi \psi_{t}^{*} \\
&+\left(1-4 i \alpha_{3}+8 \gamma\right)\left(2+|\psi|^{2}\right) .
\end{aligned}
$$

\section{APPENDIX B: POLYNOMIALS FOR THE LPD SOLUTION OF EQ. (52)}

$$
\begin{aligned}
G_{p}=3 & -T^{4}-2\left(3 T^{2}+17\right) X^{2}-6 T^{2}+32 x X-5 X^{4}, \\
H_{p}= & -2\left(T^{2}+5\right) X^{3}+\left(-T^{4}+14 T^{2}+23\right) X \\
+ & 16 x X^{2}-X^{5}-16\left(T^{2}+1\right) x, \\
D_{p}= & 36\left(4912 \gamma^{2}+456 \gamma+11\right) x^{2}+T^{6}+X^{6}+9 \\
& +3 T^{2}\left[-12(44 \gamma+1) x X+X^{4}+9\right] \\
& +6(172 \gamma+9) x X^{3}+3 T^{4}\left(X^{2}+1\right) .
\end{aligned}
$$

Here $X=2(12 \gamma+1) x$ and $T=2 t$. As we can see, our generalized approach correctly produces the previously known solutions.

\section{APPENDIX C: POLYNOMIALS FOR THE HIROTA SOLUTION OF EQ. (56)}

$$
\begin{gathered}
G_{h}=3-4 T\left[t\left(2 t^{2}+3 T w+3\right)+w\left(2 w^{2}+11\right)\right]-6\left(T^{2}+3\right) X^{2}-5 X^{4}, \\
H_{h}=X\left\{-4 T\left[t\left(2 t^{2}+3 T w-3\right)+w\left(2 w^{2}+5\right)\right]-2\left(T^{2}+1\right) X^{2}-X^{4}+15\right\}, \\
D_{h}=9+108 t^{2}+64 t^{6}+48 t^{4}+3 X^{2}\left\{4 T\left[t\left(2 t^{2}+3 T w-3\right)+w\left(2 w^{2}+5\right)\right]+33\right\}+4 w\left\{2 \left[48 t^{5}+120 t^{4} w+8 t^{3}\left(20 w^{2}-1\right)\right.\right. \\
\left.\left.+60 t^{2} w\left(2 w^{2}-1\right)+t\left(48 w^{4}-72 w^{2}+51\right)+8 w^{5}-26 w^{3}\right]+139 w\right\}+3\left(T^{2}+9\right) X^{4}+X^{6} .
\end{gathered}
$$

Here $X=2 x$ and $w=6 \alpha_{3} x$, while $T=2(t+w)$. 
[1] A. Osborne, Nonlinear Ocean Waves and the Inverse Scattering Transform (Elsevier, New York, 2010).

[2] G. P. Agrawal, Nonlinear Fiber Optics (Optics and Photonics), 4th ed. (Academic, New York, 2006), Chap. 4.

[3] H. Bailung, S. K. Sharma, and Y. Nakamura, Phys. Rev. Lett. 107, 255005 (2011).

[4] E. P. Gross, Il Nuovo Cimento 20, 454 (1961).

[5] L. P. Pitaevskii, Soviet Phys. JETP 13, 451 (1961).

[6] V. E. Zakharov and A. B. Shabat, J. Exp. Theor. Phys. 34, 62 (1972).

[7] M. J. Potasek and M. Tabor, Phys. Lett. A 154, 449 (1991).

[8] S. B. Cavalcanti, J. C. Cressoni, H. R. da Cruz, and A. S. Gouveia-Neto, Phys. Rev. A 43, 6162 (1991).

[9] Y. Kodama, A. V. Mikhailov, and S. Wabnitz, Opt. Commun. 143, 53 (1997).

[10] M. Trippenbach and Y. B. Band, Phys. Rev. A 57, 4791 (1998).

[11] R. Hirota, J. Math. Phys. 14, 805 (1973).

[12] N. Sasa and J. Satsuma, J. Phys. Soc. Jpn. 60, 409 (1991).

[13] M. Lakshmanan, K. Porsezian, and M. Daniel, Phys. Lett. A 133, 483 (1988).

[14] Yu. V. Sedletskii, J. Exp. Theor. Phys. 97, 180 (2003) [124, 200 (2003)].

[15] A. V. Slunyaev, J. Exp. Theor. Phys. 101, 926 (2005) [128, 1061 (2005)].

[16] N. Akhmediev and A. Ankiewicz, Solitons, Nonlinear Pulses and Beams (Chapman and Hall, London, 1997), See Sec. 2.7.

[17] A. Ankiewicz, J. M. Soto-Crespo, and N. Akhmediev, Phys. Rev. E 81, 046602 (2010).

[18] K. Porsezian, M. Daniel, and M. Lakshmanan, J. Math. Phys. 33, 1807 (1992).

[19] K. Porsezian, Phys. Rev. E 55, 3785 (1997).

[20] H. Q. Zhang et al., Eur. Phys. J. 72, 233 (2009).
[21] A. Ankiewicz, N. Devine, and N. Akhmediev, Phys. Lett. A 373, 3997 (2009).

[22] A. Ankiewicz, J. M. Soto-Crespo, M. A. Chowdhury, and N. Akhmediev, J. Opt. Soc. Am. B 30, 87 (2013).

[23] U. Bandelow and N. Akhmediev, Phys. Lett. A 376, 1558 (2012).

[24] Shihua Chen, Phys. Rev. E 88, 023202 (2013).

[25] D. Mihalache, L. Torner, F. Moldoveanu, N.-C. Panoiu, and N. Truta, J. Phys. A: Math. Gen. 26, L757 (1993).

[26] D. Mihalache, N.-C. Panoiu, F. Moldoveanu, and D.-M. Baboiu, J. Phys. A: Math. Gen. 27, 6177 (1994).

[27] D. Mihalache, L. Torner, F. Moldoveanu, N.-C. Panoiu, and N. Truta, Phys. Rev. E 48, 4699 (1993).

[28] G. P. Veldes, J. Borhanian, M. McKerr, V. Saxena, D. J. Frantzeskakis, and I. Kourakis, J. Opt. 15, 064003 (2013).

[29] N. Akhmediev, J. M. Dudley, D. R. Solli, and S. K. Turitsyn, J. Opt. 15, 060201 (2013).

[30] V. I. Shrira and V. V. Geogjaev, J. Eng. Math. 67, 11 (2010).

[31] D. H. Peregrine, J. Aust. Math. Soc. Appl. Math. Ser B 25, 16 (1983).

[32] L. H. Wang, K. Porsezian, and J. S. He, Phys. Rev. E 87, 053202 (2013).

[33] A. Ankiewicz, A. Chowdhury, N. Devine, and N. Akhmediev, J. Opt. 15, 064007 (2013).

[34] N. Akhmediev, A. Ankiewicz, and J. M. Soto-Crespo, Phys. Rev. E 80, 026601 (2009).

[35] A. Ankiewicz and D. J. Kedziora, Phys. Lett. A 375, 2782 (2011).

[36] T. Kano, J. Phys. Soc. Jpn. 58, 4322 (1989).

[37] Y. Kodama and A. V. Mikhailov, in Algebraic Aspects of Integrable Equations, edited by A. Fokas (Birkhauser, Boston, 1996). 